\title{
On the Bogomolov-Miyaoka-Yau Inequality for Stacky Surfaces
}

\author{
Jiun-Cheng Chen* and Hsian-Hua Tseng
}

\begin{abstract}
We discuss a generalization of the Bogomolov-Miyaoka-Yau inequality to Deligne-Mumford surfaces of general type.
\end{abstract}

\section{Introduction}

We work over $\mathbb{C}$.

For a smooth complex projective surface $S$ of general type, the Bogomolov-MiyaokaYau inequality for $S$ reads (see [9] )

$$
3 c_{2}\left(T_{S}\right) \geq c_{1}\left(T_{S}\right)^{2}
$$

Together with Noether's inequality, this puts constraints on the topology of surfaces of general types. Generalizations of (1.1) to singular surfaces and surface pairs have been found, see for example $6,7,10$.

In this paper we discuss a generalization of 1.1 to Deligne-Mumford stacks. Let $\mathcal{X}$ be a smooth proper Deligne-Mumford $\mathbb{C}$-stack of dimension 2. Let $\pi: \mathcal{X} \rightarrow X$ be the natural map to the coarse moduli space. We assume that $X$ is a projective variety. Since $\mathcal{X}$ is assumed to be smooth, it has a tangent bundle $T_{\mathcal{X}}$. A good theory of Chern classes is available for Deligne-Mumford stacks, see for example [5, 15].

Theorem 1.1. Let $\mathcal{X}$ be as above. Assume that the canonical bundle $K_{\mathcal{X}}:=\wedge^{2} T_{\mathcal{X}}^{\vee}$ is numerically effective, then

$$
3 c_{2}\left(T_{\mathcal{X}}\right) \geq c_{1}\left(T_{\mathcal{X}}\right)^{2}
$$

Certainly 1.2 takes the same shape as (1.1). A proof of 1.2 , along the lines of Miyaoka's original proof of [1.1] in [9], is given in Section 2 Section 3 contains examples of (1.2). In Section 3.2 we consider 1.2 for a class of stacks $\mathcal{X}$ with stack structures in codimension 1, recovering [6, Corollary 0.2]. In Section 3.3 we consider 1.2 for Gorenstein stacks $\mathcal{X}$ with isolated stack points, recovering [10, Corollary 1.3].

Received May 9, 2019; Accepted August 13, 2019.

Communicated by Yoichi Miyaoka.

2010 Mathematics Subject Classification. 14J29, 14A20.

Key words and phrases. Deligne-Mumford stacks, Bogomolov-Miyaoka-Yau inequality.

*Corresponding author. 
Generalizations of the Bogomolov-Miyaoka-Yau inequality to varieties with quotient singularities (i.e., orbifolds) certainly have been studied before by many authors using various approaches. References to these can be found in e.g., [6, 7]. In this paper we work in the context of Deligne-Mumford stacks. This viewpoint has the advantage that (1.2) can be proven by following Miyaoka's original arguments in [9]. Also, as discussed in Section 3. (1.2) specializes to some generalizations of the original (1.1) by straightforward and elementary means.

\section{Proof of $(1.2)$}

In this section we give a proof of 1 (1.2). Our proof is adapted from Miyaoka's original proof in $[9]$.

Let $\mathcal{X}$ be a smooth proper Deligne-Mumford stack of dimension 2. If $\mathcal{X}$ has non-trivial stack structures at generic points, then $\mathcal{X}$ is an étale gerbe over a stack with trivial generic stack structure, see for example [2, Proposition 4.6]. More precisely, there is a finite group $G$, a stack $\mathcal{X}^{\prime}$ with trivial generic stabilizers, and a morphism $f: \mathcal{X} \rightarrow \mathcal{X}^{\prime}$ realizing $\mathcal{X}$ as a $G$-gerbe over $\mathcal{X}^{\prime}$. Since $T_{\mathcal{X}}=f^{*} T_{\mathcal{X}^{\prime}}$, we see that 1.2$)$ for $\mathcal{X}$ is equivalent to 1.2 for $\mathcal{X}^{\prime}$. Therefore it suffices to consider only those $\mathcal{X}$ with stack structures in codimension $\geq 1$. For the rest of this section we assume this.

Let $\mathcal{F}$ be a locally free sheaf of rank 2 on $\mathcal{X}$. Let $\mathcal{V}:=\mathbb{P}(\mathcal{F})$ be the projectivization, with natural projection $p: \mathcal{V} \rightarrow \mathcal{X}$. Let $\mathcal{H}$ be the divisor associated to the tautological sheaf on $\mathcal{V}$.

Lemma 2.1. Assume that $\mathcal{W} \subset \mathcal{V}$ is linearly equivalent to $\mathcal{H}-p^{*} \mathcal{D}$, where $\mathcal{D} \subset \mathcal{X}$ is a divisor on $\mathcal{X}$. Then we have

$$
\mathcal{D} \cdot \operatorname{det} \mathcal{F} \leq c_{2}(\mathcal{F})+\mathcal{D}^{2}
$$

Proof. We closely follow Miyaoka's original proof [9]. Let $i: \mathcal{W} \subset \mathcal{V}$ be the inclusion morphism. Note that the composition $p \circ i: \mathcal{W} \rightarrow \mathcal{X}$ is birational by our assumption on the linear equivalence class of $\mathcal{W}$. Since resolutions can be chosen such that they are compatible with étale base change, there is a sequence of blow-ups

$$
\mu: \mathcal{V}_{s} \stackrel{\mu_{s}}{\longrightarrow} \mathcal{V}_{s-1} \rightarrow \cdots \rightarrow \mathcal{V}_{1} \stackrel{\mu_{1}}{\rightarrow} \mathcal{V}_{0}=\mathcal{V}
$$

such that the proper transform $\mathcal{W}^{\prime}$ of $\mathcal{W}$ is a smooth Deligne-Mumford stack in $\mathcal{V}_{s}$. Let $i^{\prime}: \mathcal{W}^{\prime} \subset \mathcal{V}_{s}$ and $\rho: \mathcal{W}^{\prime} \rightarrow \mathcal{X}$ be the natural maps.

Let $\mathcal{E}_{1}, \mathcal{E}_{2}, \ldots, \mathcal{E}_{s}$ be the exceptional divisors on $\mathcal{V}_{s}$. The divisor $\mathcal{W}^{\prime}$ is linearly equivalent to $\mu^{*}\left(\mathcal{H}-p^{*} \mathcal{D}\right)-\sum a_{i} \mathcal{E}_{i}$. It can be seen $]^{1}$ that the canonical bundle $K_{\mathcal{W}^{\prime}}$ satisfies $K_{\mathcal{W}^{\prime}}=$

\footnotetext{
${ }^{1}$ The argument is similar to that of $[9$, Lemma 7] and is omitted.
} 
$\rho^{*} K_{\mathcal{X}}+\sum \mathcal{C}_{i}$ where $C_{i}$ is a curve and $\rho\left(\mathcal{C}_{i}\right)=$ point. By the Hodge index theorem (for a stacky version see 8 , Theorem 3.1.3]), it follows that $\left(K_{\mathcal{W}^{\prime}}-\rho^{*} K_{\mathcal{X}}+\sum c_{i} i^{\prime *} \mathcal{E}_{i}\right)^{2} \leq 0$ for any $c_{i} \in \mathbb{R}$.

Write $K_{\mathcal{V}_{s}}=\mu^{*}\left(-2 \mathcal{H}+p^{*} K_{\mathcal{X}}+p^{*}(\operatorname{det} \mathcal{F})\right)+\sum b_{i} \mathcal{E}_{i}$. The adjunction formula implies that

$$
K_{\mathcal{W}^{\prime}}=i^{*}\left[\mu^{*}(-\mathcal{H})+(p \circ \mu)^{*}\left(K_{\mathcal{X}}+\operatorname{det} \mathcal{F}-\mathcal{D}\right)+\sum\left(b_{i}-a_{i}\right) \mathcal{E}_{i}\right] .
$$

Thus $i^{\prime *} \mu^{*}\left(-\mathcal{H}+p^{*}(\operatorname{det} \mathcal{F}-\mathcal{D})\right)^{2} \leq 0$. Set $k:=i^{\prime *} \mu^{*}\left(-\mathcal{H}+p^{*}(\operatorname{det} \mathcal{F}-\mathcal{D})\right)^{2}$. We can also compute this self-intersection number $k$ in another way:

$$
\begin{array}{rlr}
k & =\mu^{*}\left(-\mathcal{H}+p^{*}(\operatorname{det} \mathcal{F})-p^{*} \mathcal{D}\right)^{2}\left(\mu^{*} \mathcal{H}-(p \circ \mu)^{*} \mathcal{D}-\sum a_{i} \mathcal{E}_{i}\right) & \\
& =\mu^{*}\left(-\mathcal{H}+p^{*}(\operatorname{det} \mathcal{F})-p^{*} \mathcal{D}\right)^{2}\left(\mu^{*} \mathcal{H}-(p \circ \mu)^{*} \mathcal{D}\right) & \text { (since } \mathcal{E}_{i} \text { is exceptional) } \\
& =\mathcal{H}^{3}-\mathcal{H}^{2} \cdot p^{*}(\mathcal{D}-2 \operatorname{det} \mathcal{F})+\mathcal{H} \cdot\left(p^{*}(\operatorname{det} \mathcal{F})^{2}-\left(p^{*} \mathcal{D}\right)^{2}\right) &
\end{array}
$$

Using the standard relations for the intersection numbers on the projectivization of a rank 2 vector bundle, we calculate that

$$
k=c_{1}^{2}(\operatorname{det}(\mathcal{F}))-c_{2}(\mathcal{F})-(\operatorname{det} \mathcal{F})^{2}+\operatorname{det} \mathcal{F} \cdot \mathcal{D}-\mathcal{D}^{2}=-c_{2}(\mathcal{F})+\operatorname{det} \mathcal{F} \cdot \mathcal{D}-\mathcal{D}^{2}
$$

The result follows.

Let $\mathcal{O}_{\mathcal{X}}(\mathcal{D})$ be a subsheaf of $\Omega_{\mathcal{X}}^{1}$. One key observation used in Miyaoka's original proof is that the Iitaka dimension of $\mathcal{O}_{\mathcal{X}}(\mathcal{D})$ is at most 1.

Theorem 2.2. If $\mathcal{O}_{\mathcal{X}}(\mathcal{D})$ is a subsheaf of $\Omega_{\mathcal{X}}^{1}$ of a projective Deligne-Mumford stack $\mathcal{X}$, then $h^{0}\left(\mathcal{X}, \mathcal{O}_{\mathcal{X}}(n \mathcal{D})\right) \leq$ cn for some positive constant $c$ and $n \gg 0$.

The proof of Theorem 2.2 is very similar to that of [9, Theorem 2"]. Two main ingredients are needed in the proof of [9, Theorem 2"]: (1) the Riemann-Roch formula, and (2) a lemma due to de Franchis. The de Franchis lemma states that any global holomorphic differential form on a Kähler manifold or a surface is $d$-closed [9, Lemma 9]. This lemma follows essentially from Stoke's theorem. The argument still works for smooth Kähler Deligne-Mumford stacks (Kähler orbifolds) or smooth surface Deligne-Mumford stacks. The Riemann-Roch for stacks is proved in [12].

One can prove the following result using Theorem 2.2 .

Proposition 2.3. Let $\mathcal{F} \subset \Omega_{\mathcal{X}}^{1}$ be a locally free sheaf of rank 2 and assume that $\operatorname{det}(\mathcal{F})^{\otimes n}$ is generated by global sections for some $n>0$. If $\mathcal{F} \otimes \mathcal{O}_{\mathcal{X}}(-\mathcal{D})$ has a non-trivial section, then

$$
\mathcal{D} \cdot \operatorname{det}(\mathcal{F}) \leq \max \left\{c_{2}(\mathcal{F}), 0\right\}
$$


Proof. Consider $p: \mathcal{V}=\mathbb{P}(\mathcal{F}) \rightarrow \mathcal{X}$. The canonical isomorphism gives us

$$
H^{0}\left(\mathcal{X}, \mathcal{F} \otimes \mathcal{O}_{\mathcal{X}}(-\mathcal{D})\right)=H^{0}\left(\mathcal{V}, \mathcal{O}_{\mathcal{V}}\left(\mathcal{H}-p^{*} \mathcal{D}\right)\right)
$$

If $\mathcal{F} \otimes \mathcal{O}_{\mathcal{X}}(-\mathcal{D})$ has a non-trivial section, then $\left|\mathcal{H}-p^{*} \mathcal{D}\right|$ is non-empty. Pick $\mathcal{W} \in\left|\mathcal{H}-p^{*} \mathcal{D}\right|$. Decompose $\mathcal{W}$ as $\mathcal{W}=\mathcal{W}_{0}+p^{*} \mathcal{D}^{\prime}$ where $\mathcal{W}_{0}$ is effective and irreducible which is linearly equivalent to $\mathcal{H}-p^{*}\left(\mathcal{D}+\mathcal{D}^{\prime}\right)$ and $\mathcal{D}^{\prime}$ is effective. Note that $(\operatorname{det} \mathcal{F})^{\otimes n}$ is generated by global sections, so the intersection number $\mathcal{D}^{\prime} \cdot \operatorname{det}(\mathcal{F}) \geq 0$. It follows that $\mathcal{D} \cdot \operatorname{det}(\mathcal{F}) \leq$ $\left(\mathcal{D}+\mathcal{D}^{\prime}\right) \cdot \operatorname{det}(\mathcal{F})$ and it suffices to prove $\left(\mathcal{D}+\mathcal{D}^{\prime}\right) \cdot \operatorname{det}(\mathcal{F}) \leq \max \left\{c_{2}(\mathcal{F}), 0\right\}$. Set $\mathcal{D}^{\prime \prime}=\mathcal{D}+\mathcal{D}^{\prime}$ to simplify notation. By Lemma 2.1, $\mathcal{D}^{\prime \prime} \cdot \operatorname{det}(\mathcal{F}) \leq c_{2}(\mathcal{F})+\mathcal{D}^{\prime \prime} \cdot \mathcal{D}^{\prime \prime}$. Observe that $\mathcal{O}_{\mathcal{X}}\left(\mathcal{D}^{\prime \prime}\right)$ is a subsheaf of $\Omega_{\mathcal{X}}^{1}$. Indeed, the effectiveness of $\mathcal{W}_{0}$ ensures the existence of a non-trivial section of $\mathcal{F} \otimes \mathcal{O}_{\mathcal{X}}\left(-\mathcal{D}^{\prime \prime}\right)$, i.e., an injection $\mathcal{O}_{\mathcal{X}} \hookrightarrow \mathcal{F} \otimes \mathcal{O}_{\mathcal{X}}\left(-\mathcal{D}^{\prime \prime}\right)$. Twisting by $\mathcal{O}_{\mathcal{X}}\left(-\mathcal{D}^{\prime \prime}\right)$, embeds $\mathcal{O}_{\mathcal{X}}\left(-\mathcal{D}^{\prime \prime}\right)$ into $\mathcal{F} \subset \Omega_{\mathcal{X}}^{1}$. By Theorem 2.2. $\mathcal{D}^{\prime \prime}$ has Iitaka dimension at most 1 . It follows that $\mathcal{D}^{\prime \prime} \cdot \operatorname{det}(\mathcal{F}) \leq 0$ or $\mathcal{D}^{\prime \prime} \cdot \mathcal{D}^{\prime \prime} \leq 02$ This completes the proof.

Assuming $c_{2}(\mathcal{F})$ is positive for the time being, we can obtain an upper bound on $c_{2}$ provided the sheaf $\mathcal{F} \otimes \mathcal{O}_{\mathcal{X}}(-\mathcal{D})$ has no sections. This can then be used to derive a contradiction. To be more precise, one needs a modified version of Proposition 2.3, in which the condition on the sheaf $\mathcal{F} \otimes \mathcal{O}_{\mathcal{X}}(-\mathcal{D})$ having a section is replaced by the condition that some symmetric power $S^{m} \mathcal{F} \otimes \mathcal{O}_{\mathcal{X}}(-\mathcal{D})$ having a section.

Theorem 2.4. Let $\mathcal{F} \subset \Omega_{\mathcal{X}}^{1}$ be a locally free sheaf of rank 2 and assume that $\operatorname{det}(\mathcal{F})^{\otimes n}$ is generated by global sections for some $n>0$. If $S^{m} \mathcal{F} \otimes \mathcal{O}_{\mathcal{X}}(-\mathcal{D})$ has a non-trivial section, then

$$
\mathcal{D} \cdot \operatorname{det}(\mathcal{F}) \leq \max \left\{m c_{2}(\mathcal{F}), 0\right\} .
$$

The proof of Theorem 2.4 follows from Proposition 2.3 and the following easy lemma (which is analogous to [9, Lemma 11]).

Lemma 2.5. Let $p: \mathcal{V}=\mathbb{P}(\mathcal{F}) \rightarrow \mathcal{X}$ be the projective bundle of a locally free sheaf of rank 2. Let $\mathcal{W} \in\left|m \mathcal{H}-p^{*} \mathcal{D}\right|$. Then there is a surjective morphism $\beta: \mathcal{X}^{\prime} \rightarrow \mathcal{X}$ such that $\beta^{*} \mathcal{W}$ is decomposed to $\mathcal{W}_{1}+\cdots+\mathcal{W}_{m}$ where $\mathcal{W}_{i}$ is an effective divisor linear equivalent to $\mathcal{H}^{\prime}-p^{*} \mathcal{D}_{i}$.

Proof of Theorem 2.4. The following argument is taken from [9, Theorem 3]. Let $f$ be a global section of $S^{m} \mathcal{F} \otimes \mathcal{O}_{\mathcal{X}}(-\mathcal{D})$. Lemma 2.5 implies that after a suitable cover $\beta: \mathcal{Y} \rightarrow \mathcal{X}$, we can decompose $\beta^{*} f$ can be written as $f_{1} f_{2} \cdots f_{m} \in H^{0}\left(\mathcal{Y}, S^{m} \beta^{*} \mathcal{F} \otimes \mathcal{O}_{\mathcal{Y}}\left(-\beta^{*} \mathcal{D}\right)\right.$ ), where $f_{i} \in H^{0}\left(\mathcal{Y}, \beta^{*} \mathcal{F} \otimes \mathcal{O}_{\mathcal{Y}}\left(-\beta^{*} \mathcal{D}_{i}\right)\right)$ and $\left(\operatorname{det} \beta^{*} \mathcal{F}\right)^{\otimes m} \cong\left(\beta^{*} \operatorname{det}(\mathcal{F})^{\otimes m}\right)$ is generated by global sections. From Proposition 2.3 it follows that $\beta^{*} \mathcal{D}_{i} \cdot\left(\operatorname{det}\left(\beta^{*} \mathcal{F}\right)\right) \leq \max \left\{c_{2}\left(\beta^{*} \mathcal{F}\right), 0\right\}$. Summing over all $i$ 's, we have $\beta^{*} \mathcal{D} \cdot \operatorname{det}\left(\beta^{*} \mathcal{F}\right) \leq \max \left\{m c_{2}\left(\beta^{*} \mathcal{F}\right), 0\right\}$. Let $d$ be the mapping degree of $\beta$. Clearly, $\beta^{*} \mathcal{D} \cdot \operatorname{det}\left(\beta^{*} \mathcal{F}\right)=d D \cdot \operatorname{det}(\mathcal{F})$ and $c_{2}\left(\beta^{*} \mathcal{F}\right)=d \beta^{*} c_{2}(\mathcal{F})$.

\footnotetext{
${ }^{2}$ Arguing as in 9 , Lemma 10].
} 
We now come to 1.2 .

Theorem 2.6. Let $\mathcal{X}$ be a non-singular Deligne-Mumfors stack with the projective coarse space $X$ of general type and $c_{1}(\mathcal{X})$ nef. Then $c_{1}^{2}(\mathcal{X}) \leq 3 c_{2}(\mathcal{X})$ holds.

Proof. As in [9], we consider two cases: $(1) c_{1}^{2}(\mathcal{X}) \leq 2 c_{2}(\mathcal{X})$ and $(2) c_{1}^{2}(\mathcal{X})>2 c_{2}(\mathcal{X})$. The first case is obvious. For the second case, set $\alpha:=c_{2}(\mathcal{X}) / c_{1}^{2}(\mathcal{X})$. Note that $\alpha<1 / 2$. Pick $\delta>0$ sufficiently small and rational. By Theorem 2.4 applied to $\mathcal{D}=m(\alpha+\delta) K_{\mathcal{X}}$, $\mathcal{F}=\Omega_{\mathcal{X}}^{1}$, we can find a positive integer $m$ such that $m(\alpha+\delta) \in \mathbb{Z}$, and

$$
h^{0}\left(\mathcal{X}, S^{m} \Omega_{\mathcal{X}}^{1} \otimes \mathcal{O}_{\mathcal{X}}\left(-m(\alpha+\delta) K_{\mathcal{X}}\right)\right)=0 .
$$

By Serre duality for smooth projective Deligne-Mumford stacks [11, Theorem 2.22], we have

$$
h^{2}\left(\mathcal{X}, S^{m} \Omega_{\mathcal{X}}^{1} \otimes \mathcal{O}_{\mathcal{X}}\left(-m(\alpha+\delta) K_{\mathcal{X}}\right)\right)=h^{0}\left(\mathcal{X}, S^{m} \Omega_{\mathcal{X}}^{1} \otimes \mathcal{O}_{\mathcal{X}}\left(-m(1-\alpha-\delta) K_{\mathcal{X}}\right) \otimes K_{\mathcal{X}}\right)
$$

As $\alpha<1 / 2$ and $\delta$ is small, we have $1-\alpha-\delta>\alpha$. We apply Theorem 2.4 to $\mathcal{D}=$ $m(2-\alpha-\delta) K_{\mathcal{X}}, \mathcal{F}=\Omega_{\mathcal{X}}^{1}$, to get

$$
h^{2}\left(\mathcal{X}, S^{m} \Omega_{\mathcal{X}}^{1} \otimes \mathcal{O}_{\mathcal{X}}\left(-m(\alpha+\delta) K_{\mathcal{X}}\right)\right)=0
$$

Hence

$$
\chi\left(\mathcal{X}, S^{m} \Omega_{\mathcal{X}} \otimes \mathcal{O}\left(-m(\alpha+\delta) K_{\mathcal{X}}\right)\right)=-h^{1}\left(\mathcal{X}, S^{m} \Omega_{\mathcal{X}}^{1} \otimes \mathcal{O}_{\mathcal{X}}\left(-m(\alpha+\delta) K_{\mathcal{X}}\right)\right) \leq 0
$$

Note that to compute the cohomology groups of a (subsheaf of) symmetric power of a vector bundle, one can work on the the projectivized vector bundle and computing the cohomology groups of relevant line bundles. Thus

$$
0 \geq \chi\left(\mathcal{X}, S^{m} \Omega_{\mathcal{X}} \otimes \mathcal{O}\left(-m(\alpha+\delta) K_{\mathcal{X}}\right)\right)=\chi\left(\mathcal{V}, \mathcal{O}_{\mathcal{V}}\left(-m\left(\mathcal{H}-(\alpha+\delta) \pi^{*} K_{\mathcal{X}}\right)\right)\right)
$$

By Riemann-Roch for stacks [12], we have $\chi\left(\mathcal{V}, \mathcal{O}_{\mathcal{V}}\left(-m\left(\mathcal{H}-(\alpha+\delta) \pi^{*} K_{\mathcal{X}}\right)\right)\right)$ grows like $\frac{1}{6}\left(\mathcal{H}-(\alpha+\delta) \pi^{*} K_{\mathcal{X}}\right)^{3} m^{3}$ as $m \rightarrow \infty$. It implies that $\left(\mathcal{H}-(\alpha+\delta) \pi^{*} K_{\mathcal{X}}\right)^{3} \leq 0$. Taking $\delta$ to 0 , we obtain

$$
\begin{aligned}
0 \geq\left(\mathcal{H}-\alpha \pi^{*} K_{\mathcal{X}}\right)^{3} & =c_{1}^{2}(\mathcal{X})-c_{2}(\mathcal{X})-3 \alpha c_{1}^{2}(\mathcal{X})+3 \alpha^{2} c_{1}^{2}(\mathcal{X}) \\
& =\left(1-\alpha-3 \alpha+3 \alpha^{2}\right) c_{1}^{2}(\mathcal{X}) \\
& =(1-\alpha)(1-3 \alpha) c_{1}^{2}(\mathcal{X})
\end{aligned}
$$

Since $\alpha<1 / 2$ and $c_{1}^{2}(\mathcal{X})$ is non-negative, we get $1-3 \alpha \leq 0$ as desired. 


\section{Examples of 1.2}

\subsection{General discussion}

According to the main result of [4], the map $\mathcal{X} \rightarrow X$ from the stack $\mathcal{X}$ to its coarse moduli space $X$ (which we assume to be a variety with quotient singularities) can be factored as

$$
\mathcal{X} \rightarrow \mathcal{X}_{1} \rightarrow \mathcal{X}_{2} \rightarrow X,
$$

where

(1) $\mathcal{X}_{1}$ has trivial generic stabilizers;

(2) $\mathcal{X}_{2}$ is the canonical stack associated to the variety $X$ (see e.g., [3. Definition 4.4]) and has stack structures in codimension at least 2 ;

(3) $\mathcal{X} \rightarrow \mathcal{X}_{1}$ is a gerbe;

(4) $\mathcal{X}_{1} \rightarrow \mathcal{X}_{2}$ is a composition of root constructions along divisors, thus introducing codimension-1 stack structures to $\mathcal{X}_{2}$.

Since $\mathcal{X} \rightarrow \mathcal{X}_{1}$ is a gerbe, the tangent bundle of $\mathcal{X}_{1}$ pulls back to the tangent bundle of $\mathcal{X}$. So the inequality $(1.2)$ for $\mathcal{X}$ is equivalent to 1.2 for $\mathcal{X}_{1}$. Therefore when considering examples, we may restrict our attention to $\mathcal{X}$ whose stack structures are in codimension at least 1. In the rest of this section we present two examples of (1.2): the example in Section 3.2 is obtained by root constructions, and the examples in Section 3.3 are canonical stacks associated to quotient varieties. In these examples we show that 1.2 coincides with previous results.

\subsection{Codimension 1 stack structure}

We consider 1.2 for an example of stack $\mathcal{X}$ with stack structures in codimension 1.

Let $X$ be a smooth complex projective surface and $D$ a simple normal crossing $\mathbb{Q}$ divisor of the form $D=\sum_{i}\left(1-1 / r_{i}\right) D_{i}$ with $r_{i} \geq 2$ integers. Let $\mathcal{X}$ be the natural stack cover of the pair $(X, D)$. By construction the coarse moduli space of $\mathcal{X}$ is $X$. The natural map $\pi: \mathcal{X} \rightarrow X$ is an isomorphism outside $\pi^{-1}(\operatorname{Supp} D)$, which is where $\mathcal{X}$ has non-trivial stack structures. The stack $\mathcal{X}$ can be constructed from $X$ by applying root constructions along components of $D$. Furthermore we have the following formula for the canonical bundle:

$$
K_{\mathcal{X}}=\pi^{*}\left(K_{X}+D\right)
$$

We now examine 1.2 for this $\mathcal{X}$. By (3.1),

$$
c_{1}\left(T_{\mathcal{X}}\right)^{2}=c_{1}\left(K_{\mathcal{X}}\right)^{2}=\left(K_{X}+D\right)^{2} .
$$


By Gauss-Bonnet theorem for Deligne-Mumford stacks [13, Corollaire 3.44] we have

$$
c_{2}\left(T_{\mathcal{X}}\right)=\chi(\mathcal{X})
$$

the Euler characteristic of $\mathcal{X}$ as defined in [13, Definition 3.43] (note that the notation $\chi^{\text {orb }}$ is used in 13 ). Put

$$
\mathcal{D}_{i}:=\pi^{-1}\left(D_{i}\right), \quad \mathcal{D}_{i}^{\circ}:=\mathcal{D}_{i} \backslash\left(\bigcup_{j \neq i}\left(\mathcal{D}_{i} \cap \mathcal{D}_{j}\right)\right)
$$

Then we have

$$
\chi\left(\mathcal{X} \backslash \pi^{-1}(\operatorname{Supp} D)\right)=\chi(\mathcal{X})-\sum_{i} \chi\left(\mathcal{D}_{i}^{\circ}\right)-\sum_{p \in \mathcal{D}_{i} \cap \mathcal{D}_{j}} \chi(p)
$$

Similarly, put $D_{i}^{\circ}=D_{i} \backslash\left(\bigcup_{j \neq i}\left(D_{i} \cap D_{j}\right)\right)$, we have

$$
\chi(X \backslash \operatorname{Supp} D)=\chi(X)-\sum_{i} \chi\left(D_{i}^{\circ}\right)-\sum_{\bar{p} \in D_{i} \cap D_{j}} \chi(\bar{p}) .
$$

Since $\mathcal{X} \backslash \pi^{-1}(\operatorname{Supp} D) \simeq X \backslash \operatorname{Supp} D$, we have $\chi\left(\mathcal{X} \backslash \pi^{-1}(\operatorname{Supp} D)\right)=\chi(X \backslash \operatorname{Supp} D)$. Equivalently,

$$
\chi(\mathcal{X})=\chi(X)-\sum_{i} \chi\left(D_{i}^{\circ}\right)-\sum_{\bar{p} \in D_{i} \cap D_{j}} \chi(\bar{p})+\sum_{i} \chi\left(\mathcal{D}_{i}^{\circ}\right)+\sum_{p \in \mathcal{D}_{i} \cap \mathcal{D}_{j}} \chi(p) .
$$

Since the map $\mathcal{D}_{i}^{\circ} \rightarrow D_{i}^{\circ}$ is of degree $1 / r_{i}$ and the map $\mathcal{D}_{i} \cap \mathcal{D}_{j} \rightarrow D_{i} \cap D_{j}$ is of degree $1 /\left(r_{i} r_{j}\right)$, we have

$$
\chi\left(\mathcal{D}_{i}\right)=\frac{1}{r_{i}} \chi\left(D_{i}\right), \quad \chi\left(\mathcal{D}_{i} \cap \mathcal{D}_{j}\right)=\frac{1}{r_{i} r_{j}} \chi\left(D_{i} \cap D_{j}\right)
$$

This implies that

$$
\chi(\mathcal{X})=\chi(X)-\sum_{i}\left(1-\frac{1}{r_{i}}\right) \chi\left(D_{i}^{\circ}\right)+\sum_{\bar{p} \in D_{i} \cap D_{j}}\left(\frac{1}{r_{i} r_{j}}-1\right)
$$

By 7, Theorem 8.7], for $\bar{p} \in D_{i} \cap D_{j}$ the local orbifold Euler number of the pair $(X, D)$ at $\bar{p}$ is given by $e_{\text {orb }}(\bar{p} ; X, D)=1 /\left(r_{i} r_{j}\right)$. Together with 3.2 this implies that $\chi(\mathcal{X})$ coincides with the orbifold Euler number $e_{\mathrm{orb}}(X, D)$ of the pair $(X, D)$, as defined in [7]. Thus if $K_{\mathcal{X}}$ is numerically effective, then 1.2 is equivalent to [7, Theorem 0.1$]$ applied to the pair $(X, D)$. 


\subsection{Codimension 2 stack structure}

Let $\mathcal{X}$ be a smooth proper Deligne-Mumford $\mathbb{C}$-stack of dimension 2 with isolated stack structures. Let $\pi: \mathcal{X} \rightarrow X$ be the natural map to the coarse moduli space $X$. Let $p_{1}, p_{2}, \ldots, p_{k} \in \mathcal{X}$ be the stacky points. Suppose that $\mathcal{X}$ is Gorenstein, i.e., each stacky point $p_{i}$ has a neighborhood $p_{i} \in U_{i} \subset \mathcal{X}$ of the form $U_{i} \simeq\left[\mathbb{C}^{2} / G_{i}\right]$ with $G_{i} \subset \mathrm{SU}(2)$ a finite subgroup, identifying $p_{i}$ with $\left[0 / G_{i}\right] \in\left[\mathbb{C}^{2} / G_{i}\right]$. It is a standard fact that the coarse moduli space $X$ is a projective surface with canonical singularities.

Suppose further that $K_{\mathcal{X}}$ is numerically effective. We consider $(1.2)$ for such $\mathcal{X}$.

By assumption we have $K_{\mathcal{X}}=\pi^{*} K_{X}$. Thus

$$
c_{1}\left(T_{\mathcal{X}}\right)^{2}=c_{1}\left(K_{\mathcal{X}}\right)^{2}=c_{1}\left(K_{X}\right)^{2} .
$$

We now consider the term $c_{2}\left(T_{\mathcal{X}}\right)$. The first step is to consider $\chi\left(\mathcal{O}_{\mathcal{X}}\right)$ by using RiemannRoch theorem for stacks [12,13. We follow [14, Appendix A] for the presentation of the Riemann-Roch theorem. We have

$$
\chi\left(\mathcal{O}_{\mathcal{X}}\right)=\int_{I \mathcal{X}} \widetilde{\operatorname{ch}}\left(\mathcal{O}_{\mathcal{X}}\right) \widetilde{\mathrm{Td}}\left(T_{\mathcal{X}}\right)
$$

Here $I \mathcal{X}$ is the inertia stack of $\mathcal{X}$. By our assumption on $\mathcal{X}$, we have the following description of $I \mathcal{X}$ :

$$
I \mathcal{X}=\mathcal{X} \cup \bigcup_{i=1}^{k}\left(I p_{i} \backslash p_{i}\right)
$$

Here the term $I p_{i} \backslash p_{i}$ is the inertia stack of $p_{i} \simeq B G_{i}$ with the main component removed, namely

$$
I p_{i} \backslash p_{i} \simeq \bigcup_{(g) \neq(1): \text { conjugacy class of } G_{i}} B C_{G_{i}}(g) .
$$

By the definition of the Chern character $\widetilde{c h}$, we have $\widetilde{c h}\left(\mathcal{O}_{\mathcal{X}}\right)=1$ on every component of $I \mathcal{X}$. Hence

$$
\chi\left(\mathcal{O}_{\mathcal{X}}\right)=\int_{I \mathcal{X}} \widetilde{\operatorname{Td}}\left(T_{\mathcal{X}}\right)=\left.\int_{\mathcal{X}} \widetilde{\operatorname{Td}}\left(T_{\mathcal{X}}\right)\right|_{\mathcal{X}}+\left.\sum_{i=1}^{k} \int_{I p_{i} \backslash p_{i}} \widetilde{\operatorname{Td}}\left(T_{\mathcal{X}}\right)\right|_{I p_{i} \backslash p_{i}} .
$$

Note that $\left.\widetilde{T d}\left(T_{\mathcal{X}}\right)\right|_{\mathcal{X}}=\operatorname{Td}\left(T_{\mathcal{X}}\right)$, and we only need its degree 2 component. Hence

$$
\left.\int_{\mathcal{X}} \widetilde{\operatorname{Td}}\left(T_{\mathcal{X}}\right)\right|_{\mathcal{X}}=\frac{1}{12} \int_{\mathcal{X}}\left(c_{2}\left(T_{\mathcal{X}}\right)+c_{1}\left(T_{\mathcal{X}}\right)^{2}\right) .
$$

The contribution coming from $I p_{i} \backslash p_{i}$ can be also evaluated.

Lemma 3.1. Let $E_{i}$ be the exceptional divisor of the minimal resolution of $\mathbb{C}^{2} / G_{i}$. Then

$$
\left.\int_{I p_{i} \backslash p_{i}} \widetilde{\operatorname{Td}}\left(T_{\mathcal{X}}\right)\right|_{I p_{i} \backslash p_{i}}=\frac{1}{12}\left(\chi\left(E_{i}\right)-\frac{1}{\left|G_{i}\right|}\right) .
$$


An elementary proof of this lemma is given in the next section. Next, we reinterpret the term $\chi\left(\mathcal{O}_{\mathcal{X}}\right)$. By definition, $\chi\left(\mathcal{O}_{\mathcal{X}}\right):=\sum_{l \geq 0}(-1)^{l} \operatorname{dim} H^{l}\left(\mathcal{X}, \mathcal{O}_{\mathcal{X}}\right)$. Since $\pi_{*} \mathcal{O}_{\mathcal{X}}=\mathcal{O}_{X}$ (see e.g., 11, Theorem 2.2.1]), we have $H^{l}\left(\mathcal{X}, \mathcal{O}_{\mathcal{X}}\right)=H^{l}\left(X, \mathcal{O}_{X}\right)$ and

$$
\chi\left(\mathcal{O}_{\mathcal{X}}\right)=\chi\left(\mathcal{O}_{X}\right)
$$

Combining (3.3), (3.4), 3.5), and Lemma 3.1, we obtain the following expression of $c_{2}\left(T_{\mathcal{X}}\right)$

$$
\int_{\mathcal{X}} c_{2}\left(T_{\mathcal{X}}\right)=12 \chi\left(\mathcal{O}_{X}\right)-\int_{\mathcal{X}} c_{1}\left(T_{\mathcal{X}}\right)^{2}-\sum_{i=1}^{k}\left(\chi\left(E_{i}\right)-\frac{1}{\left|G_{i}\right|}\right) .
$$

Using this, we see that in the present situation, $(1.2)$ is equivalent to

$$
12 \chi\left(\mathcal{O}_{X}\right) \geq \frac{4}{3} c_{1}\left(K_{X}\right)^{2}+\sum_{i=1}^{k}\left(\chi\left(E_{i}\right)-\frac{1}{\left|G_{i}\right|}\right) .
$$

On the other hand, it is clear that (3.6) is a special case of [10, Corollary 1.3].

\section{Proof of Lemma 3.1}

In this section we prove Lemma 3.1. By our assumption on $\mathcal{X}$, for $g \in G_{i}$, the $g$-action on the tangent space $T_{p_{i}} \mathcal{X}$ has two eigenvalues $\xi_{g}$ and $\xi_{g}^{-1}$, where $\xi_{g}$ is a certain root of unity. By the definition of $\widetilde{T d}\left(T_{\mathcal{X}}\right)$ we have

$$
\left.\int_{I p_{i} \backslash p_{i}} \widetilde{\operatorname{Td}}\left(T_{\mathcal{X}}\right)\right|_{I p_{i} \backslash p_{i}}=\sum_{(g) \neq(1): \text { conjugacy class of } G_{i}} \frac{1}{\left|C_{G_{i}}(g)\right|} \frac{1}{2-\xi_{g}-\xi_{g}^{-1}} .
$$

We now evaluate 4.1) using the $A D E$ classification of $\mathbb{C}^{2} / G_{i}$.

\subsection{Type A}

If $\mathbb{C}^{2} / G_{i}$ is of type $A_{n-1}$, then $G_{i} \simeq \mathbb{Z}_{n}$ and the action on $\mathbb{C}^{2}$ is given as follows. If we identify $\mathbb{Z}_{n}$ with the group of $n$-th roots of 1 , then an element $\xi \in \mathbb{Z}_{n}$ acts on $\mathbb{C}^{2}$ via the matrix

$$
\left(\begin{array}{cc}
\xi & 0 \\
0 & \xi^{-1}
\end{array}\right)
$$

It follows that 4.1 is given by

$$
\frac{1}{n} \sum_{l=1}^{n-1} \frac{1}{2-\exp (2 \pi \sqrt{-1} l / n)-\exp (2 \pi \sqrt{-1} l / n)^{-1}} .
$$

By [8, Lemma 3.3.2.1], 4.2 is equal to

$$
\frac{n^{2}-1}{12 n}=\frac{1}{12}\left(n-\frac{1}{n}\right)
$$


Since the exceptional divisor of the minimal resolution of $\mathbb{C}^{2} / \mathbb{Z}_{n}$ is a chain of $(n-1)$ copies of $\mathbb{C P}^{1}$, its Euler characteristic is $n$. This proves Lemma 3.1 in type A case.

\subsection{Type D}

If $\mathbb{C}^{2} / G_{i}$ is of type $D_{n+2}$ (here $n \geq 2$ ), then $G_{i}$ is isomorphic to the binary dihedral group Dic $_{n}$. The group Dic ${ }_{n}$ is of order $4 n$ and may be presented as follows:

$$
\operatorname{Dic}_{n}=\left\langle a, x \mid a^{2 n}=1, x^{2}=a^{n}, x^{-1} a x=a^{-1}\right\rangle .
$$

The action of $\mathrm{Dic}_{n}$ on $\mathbb{C}^{2}$ is given as follows:

$$
a \mapsto\left(\begin{array}{cc}
\exp (\pi \sqrt{-1} / n) & 0 \\
0 & \exp (-\pi \sqrt{-1} / n)
\end{array}\right), \quad x \mapsto\left(\begin{array}{cc}
0 & 1 \\
-1 & 0
\end{array}\right) .
$$

An elementary calculation shows that the conjugacy classes of $\mathrm{Dic}_{n}$ and the orders of their centralizer subgroups are given as follows:

$\{1\}, \quad\left\{a^{n}\right\}$, $($ order of centralizer group $=4 n)$

$\left\{a^{l}, a^{-l}\right\}, \quad 1 \leq l \leq n-1$, (order of centralizer group $=2 n$ ) $\left\{x a, x a^{3}, x a^{5}, \ldots, x a^{2 n-1}\right\}, \quad\left\{x, x a^{2}, x a^{4}, \ldots, x a^{2 n-2}\right\}, \quad($ order of centralizer group $=4)$. Using (4.3) and (4.4) it is easy to identify the contribution from each conjugacy class. It follows that (4.1) is given by

$$
\frac{1}{2 n} \sum_{k=1}^{n-1} \frac{1}{2-\exp (\pi \sqrt{-1} k / n)-\exp (\pi \sqrt{-1} k / n)^{-1}}+\frac{1}{16 n}+\frac{1}{8}+\frac{1}{8} .
$$

We need to evaluate the first sum above. Again by [8, Lemma 3.3.2.1], we have

$$
\begin{aligned}
\frac{(2 n)^{2}-1}{12}= & \sum_{k=1}^{2 n-1} \frac{1}{2-\exp (2 \pi \sqrt{-1} k /(2 n))-\exp (2 \pi \sqrt{-1} k /(2 n))^{-1}} \\
= & \sum_{k=1}^{n-1} \frac{1}{2-\exp (\pi \sqrt{-1} k / n)-\exp (\pi \sqrt{-1} k / n)^{-1}}+\frac{1}{4} \\
& +\sum_{k=1}^{n-1} \frac{1}{2-\exp (2 \pi \sqrt{-1}(n+k) /(2 n))-\exp (2 \pi \sqrt{-1}(n+k) /(2 n))^{-1}} .
\end{aligned}
$$

Note that

$$
\begin{aligned}
& 2-\exp (2 \pi \sqrt{-1}(n+k) /(2 n))-\exp (2 \pi \sqrt{-1}(n+k) /(2 n))^{-1} \\
&= 2+\exp (\pi \sqrt{-1} k / n)+\exp (\pi \sqrt{-1} k / n)^{-1} \\
&= 2+2 \cos (\pi k / n)=4 \cos ^{2}(\pi k /(2 n))=4 \sin ^{2}(\pi(k+n) /(2 n)), \\
& 2-\exp (\pi \sqrt{-1} k / n)-\exp (\pi \sqrt{-1} k / n)^{-1}=2-2 \cos (\pi k / n)=4 \sin ^{2}(\pi k /(2 n)) .
\end{aligned}
$$


Since $\sin (\pi(k+n) /(2 n))=-\sin (\pi(k-n) /(2 n))$, we see that

$$
\begin{aligned}
& \sum_{k=1}^{n-1} \frac{1}{2-\exp (\pi \sqrt{-1} k / n)-\exp (\pi \sqrt{-1} k / n)^{-1}} \\
= & \sum_{k=1}^{n-1} \frac{1}{2-\exp (2 \pi \sqrt{-1}(n+k) /(2 n))-\exp (2 \pi \sqrt{-1}(n+k) /(2 n))^{-1}},
\end{aligned}
$$

from which it follows that

$$
2 \sum_{k=1}^{n-1} \frac{1}{2-\exp (\pi \sqrt{-1} k / n)-\exp (\pi \sqrt{-1} k / n)^{-1}}+\frac{1}{4}=\frac{(2 n)^{2}-1}{12}
$$

This shows that

$$
\sum_{k=1}^{n-1} \frac{1}{2-\exp (\pi \sqrt{-1} k / n)-\exp (\pi \sqrt{-1} k / n)^{-1}}=\frac{n^{2}-1}{6}
$$

and (4.1) is given by

$$
\frac{n^{2}-1}{12 n}+\frac{1}{16 n}+\frac{1}{8}+\frac{1}{8}=\frac{1}{12}\left(n+3-\frac{1}{4 n}\right)
$$

Since the exceptional divisor of the minimal resolution of $\mathbb{C}^{2} / \mathrm{Dic}_{n}$ is a tree of $\mathbb{C P}^{1}$ whose dual graph is the Dynkin diagram $D_{n+2}$, its Euler characteristic is $n+3$ and Lemma 3.1 is proved in this case.

\subsection{Type E}

If $\mathbb{C}^{2} / G_{i}$ is of type $E$, then there are three possibilities: $E_{6}, E_{7}, E_{8}$. The group $G_{i}$ is isomorphic to the binary tetrahedral group (for $E_{6}$ ), the binary octahedral group (for $E_{7}$ ), or the binary icosahedral group (for $E_{8}$ ). In each case the group and its action on $\mathbb{C}^{2}$ can be explicitly described, and Lemma 3.1 can be proved by computing 4.1) using this information. We work out the details for $E_{6}$ and leave the other two cases to the reader.

In the $E_{6}$ case, the group $G_{i}$ is isomorphic to the binary tetrahedral group $2 T$. This group is of order 24 and its elements can be identified with the following quaternion numbers:

$$
\frac{1}{2}( \pm 1 \pm i \pm j \pm k), \quad \pm i, \quad \pm j, \quad \pm k, \quad \pm 1
$$

The group $2 T$ has 7 conjugacy classes: 


\begin{tabular}{|c|c|c|c|c|}
\hline $\begin{array}{c}\text { Conjugacy } \\
\text { class }\end{array}$ & $(1)$ & $(-1)$ & $(i)$ & $\left(\frac{1}{2}(1+i+j+k)\right)$ \\
\hline Size & 1 & 1 & 6 & 4 \\
\hline $\begin{array}{c}\text { Conjugacy } \\
\text { class }\end{array}$ & $\left(\frac{1}{2}(1+i+j-k)\right)$ & $\left(\frac{1}{2}(-1+i+j+k)\right)$ & $\left(\frac{1}{2}(-1+i+j-k)\right)$ & \\
\hline Size & 4 & 4 & 4 & \\
\hline
\end{tabular}

The action of $2 T$ on $\mathbb{C}^{2}$ can be described using the following identification

$$
x+y i+z j+w k \mapsto\left(\begin{array}{cc}
x+y i & z+w i \\
-z+w i & x-y i
\end{array}\right) .
$$

Now it is straightforward to see that 4.1 is given by

$$
\begin{aligned}
& \frac{1}{24} \frac{1}{2-(-2)}+\frac{1}{4} \frac{1}{2-0}+\frac{1}{6} \frac{1}{2-1}+\frac{1}{6} \frac{1}{2-1}+\frac{1}{6} \frac{1}{2-(-1)}+\frac{1}{6} \frac{1}{2-(-1)} \\
= & \frac{167}{288}=\frac{1}{12}\left(7-\frac{1}{24}\right) .
\end{aligned}
$$

Since 7 is the Euler characteristic of the exceptional divisor of the minimal resolution of $\mathbb{C}^{2} / 2 T$, the result follows.

\section{Acknowledgments}

J.-C. Chen is a Golden-Jade Fellow of Kenda Foundation, Taiwan. He is supported in part by National Science Council and National Center for Theoretical Sciences, Taiwan. H.-H. Tseng is supported in part by NSF grant DMS-1047777 and Simons Foundation Collaboration Grant.

\section{References}

[1] D. Abramovich and A. Vistoli, Compactifying the space of stable maps, J. Amer. Math. Soc. 15 (2002), no. 1, 27-75.

[2] K. Behrend and B. Noohi, Uniformization of Deligne-Mumford curves, J. Reine Angew. Math. 599 (2006), 111-153.

[3] B. Fantechi, E. Mann and F. Nironi, Smooth toric Deligne-Mumford stacks, J. Reine Angew. Math. 648 (2010), 201-244.

[4] A. Geraschenko and M. Satriano, A "bottom up" characterization of smooth DeligneMumford stacks, Int. Math. Res. Not. IMRN 2017 (2017), no. 21, 6469-6483. 
[5] A. Kresch, Cycle groups for Artin stacks, Invent. Math. 138 (1999), no. 3, 495-536.

[6] A. Langer, The Bogomolov-Miyaoka-Yau inequality for log canonical surfaces, J. London Math. Soc. (2) 64 (2001), no. 2, 327-343.

[7] _ Logarithmic orbifold Euler numbers of surfaces with applications, Proc. London Math. Soc. (3) 86 (2003), no. 2, 358-396.

[8] M. Lieblich, Moduli of twisted orbifold sheaves, Adv. Math. 226 (2011), no. 5, 41454182.

[9] Y. Miyaoka, On the Chern numbers of surfaces of general type, Invent. Math. 42 (1977), 225-237.

[10] _ The maximal number of quotient singularities on surfaces with given numerical invariants, Math. Ann. 268 (1984), no. 2, 159-171.

[11] F. Nironi, Grothendieck duality for Deligne-Mumford stacks, arXiv:0811.1955.

[12] B. Toen, Théorèmes de Riemann-Roch pour les champs de Deligne-Mumford, KTheory 18 (1999), no. 1, 33-76.

[13] _ K-theory and cohomology of algebraic stacks: Riemann-Roch theorems, Dmodules and GAGA theorems, arXiv:math/9908097.

[14] H.-H. Tseng, Orbifold quantum Riemann-Roch, Lefschetz and Serre, Geom. Topol. 14 (2010), no. 1, 1-81.

[15] A. Vistoli, Intersection theory on algebraic stacks and on their moduli spaces, Invent. Math. 97 (1989), no. 3, 613-670.

Jiun-Cheng Chen

Department of Mathematics, Third General Building, National Tsing Hua University, No. 101 Sec 2 Kuang Fu Road, Hsinchu, 30043, Taiwan

E-mail address: jcchen@math.nthu.edu.tw, jcchenster@gmail.com

Hsian-Hua Tseng

Department of Mathematics, Ohio State University, 100 Math Tower, 231 West 18th Ave. Columbus OH 43210, USA

E-mail address: hhtseng@math.ohio-state.edu 